\title{
Interactions of Andronov-Hopf and Bogdanov-Takens Bifurcations
}

\author{
W. F. Langford and K. Zhan \\ Department of Mathematics and Statistics \\ University of Guelph \\ Guelph, Ontario, Canada N1G 2W1
}

June 5, 1998

\begin{abstract}
A codimension-three bifurcation, characterized by a pair of purely imaginary eigenvalues and a nonsemisimple double zero eigenvalue, arises in the study of a pair of weakly coupled nonlinear oscillators with $\mathbb{Z}_{2} \oplus \mathbb{Z}_{2}$ symmetry. The methodology is based on Arnold's ideas of versal deformations of matrices for the linear analysis, and Poincaré normal forms for the nonlinear analysis of the system. Bifurcation submanifolds of codimension one and two are identified in the parameter space. Many different classes of solutions are determined in the state space, including equilibria, limit cycles, invariant tori and the possibility of homoclinic chaos. As an application, a mechanism for energy transfer between two widely-spaced oscillation modes without strong resonance is identified.
\end{abstract}

\section{Introduction}

This study is motivated by a pair of weakly-coupled nonlinear oscillators, modeled by second-order differential equations of the general form

$$
\begin{aligned}
& \ddot{x}+\omega_{1}^{2} x+f_{1}(x, \dot{x}, y, \dot{y}, \mu)=0 \\
& \ddot{y}+\omega_{2}^{2} y+f_{2}(x, \dot{x}, y, \dot{y}, \mu)=0 .
\end{aligned}
$$


Here $f_{1}$ and $f_{2}$ are holomorphic functions of the state variables $x, \dot{x}, y, \dot{y}$ and parameters $\mu \in \mathbb{R}^{k}$. These functions are "higher order" in the sense that they contain only quadratic and higher order terms in these variables, and $f_{j}(0,0,0,0, \mu) \equiv 0, j=1,2$. The variables and parameters are all assumed to be small. The parameters $\mu$ represent physically relevant coefficients such as damping, detuning and the coupling between the oscillators; the role of these parameters will be clarified further below. In (1), the natural frequencies of the two oscillators, when linearized at $(x, \dot{x}, y, \dot{y})=(0,0,0,0)$, are given by $\omega_{1,2} \geq 0$. Systems of the form (1) occur frequently in the study of mechanical systems, electrical circuits, the biological sciences and elsewhere.

A major concern in the study of coupled oscillators is the understanding and control of resonance phenomena. V.I. Arnold has made influential contributions to the general theory of resonances, for example $[2,5,6]$. The ratio of the natural frequencies plays a leading role in determining resonances in the system (1) of two oscillators. The cases $\frac{\omega_{1}}{\omega_{2}}=\frac{p}{q}$, where $p$ and $q$ are small positive integers, $p+q \leq 5$, are called strong resonances. The remaining rational cases are called weak resonances, and cases with the ratio $\frac{\omega_{1}}{\omega_{2}}$ irrational are called nonresonant. As the names suggest, coupled oscillators normally interact very strongly at or near strong resonances, and interact weakly, if at all, in the other cases. This may be explained by the fact that in the Poincaré normal form corresponding to these coupled oscillator equations, terms of degree $p+q$ in the state variables appear. These are called resonant terms. Locally, these terms have a strong influence when $p+q$ is small and their influence decreases as $p+q$ increases.

This paper addresses the case of coupled oscillators (1) with natural frequencies which are widely separated; we assume $\omega_{1}$ very small and $\omega_{2}$ very large, relative to each other. On the basis of the above classification, this would not be considered a case of strong resonance. To fix magnitudes, rescale time in $(1)$ so that $\omega_{2}=1$. Then our assumption is $\omega_{1} \rightarrow 0$. Equivalently, we consider natural frequencies at or near the ratio $\frac{\omega_{1}}{\omega_{2}}=\frac{0}{1}$. Therefore, we may refer to this as the case of $0: 1$ resonance.

In a series of papers $[28,29,30]$, Nayfeh and coworkers have investigated a system of two weakly coupled oscillators with widely separated frequencies similar to (1), experimentally and analytically. They point out an interesting paradox. In both mechanical experiments and a mathematical model, when the system is excited near a high natural frequency, large low-frequency responses accompanied by slow modulations of the amplitude and phase of the high-frequency mode are observed. Thus, they found that there is a strong 
interaction between these two modes, and a transfer of energy from high to low frequency modes, even though by the above classification it is not a case of strong resonance.

In this paper, system (1) is viewed as a perturbation of a singular system with a bifurcation of codimension three. The low frequency mode, in the limit $\omega_{1} \rightarrow 0$, is a Bogdanov-Takens bifurcation [8, 31]. This bifurcation has codimension two. The high frequency mode is close to a classical AndronovHopf bifurcation [1, 13] which has codimension one. We verify that the combined singular system has codimension three, using the method of Arnold [3], and then investigate via Poincaré normal forms the interactions of these two classical bifurcations.

Cases of bifurcations involving a simple zero eigenvalue with a pair of purely imaginary eigenvalues (and no other eigenvalues with zero real part) have been studied by Arnold [5], Langford [19, 20], Guckenheimer and Holmes [14], Khazin and Shnol [18], Chow et al. [10] and others cited in these references. Less is known about the case of a nonsemisimple double zero eigenvalue together with an imaginary pair. Pioneering analytical results and applications have been presented by Moson [25, 26], Yu and Huseyin [33], Geogiou et al. [11], Nagata and Namachchivaya [27], and Nayfeh et al. $[28,29,30]$.

To fix ideas, throughout this paper it is assumed that each of the oscillators in (1) has an odd reflectional symmetry about the rest position. We extend this symmetry to the coupled system by assuming that the system commutes with the $\mathbb{Z}_{2} \oplus \mathbb{Z}_{2}$ symmetry group generated by two reflection operators

$$
\begin{aligned}
& f_{1}(-x,-\dot{x}, y, \dot{y})=-f_{1}(x, \dot{x}, y, \dot{y}) \\
& f_{2}(-x,-\dot{x}, y, \dot{y})=f_{2}(x, \dot{x}, y, \dot{y}) \\
& f_{1}(x, \dot{x},-y,-\dot{y})=f_{1}(x, \dot{x}, y, \dot{y}) \\
& f_{2}(x, \dot{x},-y,-\dot{y})=-f_{2}(x, \dot{x}, y, \dot{y}) \text {. }
\end{aligned}
$$

This symmetry is very common in applications; for example, the classical pendulum equation, Duffing's equation and Van der Pol's equation all have odd symmetry, as do the examples in [27, 28, 29, 30]. The symmetry (2) forbids linear coupling terms (i.e. linear $x, \dot{x}$ terms in the $\ddot{y}$-equation or viceversa) but permits nonlinear coupling (such as $x^{2} y$ in the $\ddot{y}$-equation).

Before beginning to analyze (1), we rewrite it with the linear part in a 
more explicit form.

$$
\begin{array}{r}
\ddot{x}+\delta_{1} \dot{x}+\Omega x+\hat{f}_{1}(x, \dot{x}, y, \dot{y}, \mu)=0 \\
\ddot{y}+\delta_{2} \dot{y}+y+\hat{f}_{2}(x, \dot{x}, y, \dot{y}, \mu)=0
\end{array}
$$

Here, $\delta_{1}, \delta_{2}$ are linear damping parameters and $\Omega=\left(\omega_{1} / \omega_{2}\right)^{2}$ is a small parameter which is now allowed to be negative or zero; therefore it is better though of as representing a restoring force rather than a frequency ratio. In the engineering literature, a change in sign of $\Omega$ from positive to negative is associated with a transition from "flutter" to "divergence". These three parameters are assumed to be small, and they represent all possible linear coefficients in the system consistent with the symmetries (2), after rescaling. (Recall that $t$ has been rescaled so that $\omega_{2}=1$ ). Since the functions $\hat{f}_{j}$ commute with the two reflection operators (2), they are cubic or higher order in the state variables.

With the definition

$$
\left(\begin{array}{c}
x \\
\dot{x} \\
y \\
\dot{y}
\end{array}\right) \equiv\left(\begin{array}{l}
y_{1} \\
y_{2} \\
y_{3} \\
y_{4}
\end{array}\right) \equiv \mathbf{y}
$$

system (3) can be written as the following system of first order equations, with linear part which is a perturbation of a matrix $\mathbf{A}_{0}$ in real Jordan form.

$$
\dot{\mathbf{y}}=\left[\mathbf{A}_{0}+\mathbf{C}(\hat{\mu})\right] \mathbf{y}+\hat{\mathbf{f}}
$$

where

$$
\mathbf{A}_{0}=\left(\begin{array}{cccc}
0 & 1 & 0 & 0 \\
0 & 0 & 0 & 0 \\
0 & 0 & 0 & 1 \\
0 & 0 & -1 & 0
\end{array}\right), \mathbf{C}(\hat{\mu})=\left(\begin{array}{cccc}
0 & 0 & 0 & 0 \\
-\Omega & -\delta_{1} & 0 & 0 \\
0 & 0 & 0 & 0 \\
0 & 0 & 0 & -\delta_{2}
\end{array}\right), \hat{\mathbf{f}}=\left(\begin{array}{c}
0 \\
-\hat{f}_{1} \\
0 \\
-\hat{f}_{2}
\end{array}\right)
$$

Here $\mathbf{C}(\hat{\mu})$ is the linear perturbation matrix, and $\hat{\mu}=\left(\delta_{1}, \delta_{2}, \Omega\right)$ represents the three physical parameters in the system.

We may simplify notation by introducing a mixed set of real/complex state variables with $\left(y_{1}, y_{2}\right) \in \mathbb{R}^{2}$ defined as above, and $z \in \mathbb{C}$ defined by

$$
z=y_{3}-i y_{4}, \bar{z}=y_{3}+i y_{4}
$$


(The reason for the nonstandard choice of signs of the imaginary parts of $z, \bar{z}$ is the following. In the phase plane of a second order harmonic oscillator equation, the coordinate axes are normally chosen to be $(x, y)=(x, \dot{x})$, which forces oscillations about the origin to have a clockwise orientation. In the complex plane, the convention is that a counterclockwise rotation is positive, corresponding to the differential equation $\dot{z}=i z$. The transformation (6) effects this reversal of orientation, between the real and complex phase planes.) Then system (4) is transformed to the systen

$$
\begin{aligned}
\dot{y}_{1} & =y_{2} \\
\dot{y}_{2} & =-\Omega y_{1}-\delta_{1} y_{2}-\hat{f}_{1} \\
\dot{z} & =\left(i-\frac{1}{2} \delta_{2}\right) z+\frac{1}{2} \delta_{2} \bar{z}+i \hat{f}_{2}
\end{aligned}
$$

together with a fourth equation for $\overline{\bar{z}}$ obtained by conjugation of the equation for $\dot{z}$ given here.

The matrix $\mathbf{A}_{0}$ in (4) is recognized as being in real Jordan form, with the four eigenvalues $0^{2}$ (nonsemisimple) and $\pm i$ (imaginary pair). The corresponding differential equations (4) for small amplitudes represent a peturbation of a system of two independent oscillators, one with coordinates $(x, \dot{x})$ and natural frequency $\omega_{1} \approx 0$, and the other with coordinates $(y, \dot{y})$ and natural frequency $\omega_{2}=1$. However, the results of this paper apply more generally than this. Consider any parametrized two degree-of-freedom system, which may have coefficient matrix far from $\mathbf{A}_{0}$; but suppose that, for some value of the parameters, the linearization at an equilibrium has eigenvalues $\left\{0^{2}, \pm i\right\}$ (after rescaling time). Then the linear part can be transformed to $\mathbf{A}_{0}$ by a linear change of coordinates (similarity transformation), which may be far from the identity. In this case, the state variables in (4) are the normal mode coordinates of the original system, and the symmetries (2) are symmetries of the normal modes. The state variables in this paper should be understood in that light.

Even more generally, the results of this paper apply to systems of ordinary differential equations of dimension higher than four, and to classes of infinite dimensional partial differential equations or functional differential equations, satisfying appropriate spectral conditions. If the linearization at an equilibrium solution satisfies standard compactness and hyperbolicity assumptions on the linear operator and its spectrum, then the Center Manifold Theorem applies. In this case, if the spectrum contains the eigenvalues $\left\{0^{2}, \pm i\right\}$, and the complementary spectrum is bounded away from the imaginary axis 
in the left half-plane, then the study of long-time dynamics of the full system reduces to the four dimensional system considered here. Such higher dimensional generalizations will not be pursued further here.

The paper is organized as follows. In the next section, a versal deformation of the linear part of the singular vector field is obtained, using ideas of Arnold, and the codimension is computed to be three. Section 3 presents the Poincaré normal form for this system. The original four-dimensional system is reduced to a three-dimensional one, exploiting the $S^{1}$ normal form symmetry arising from the Andronov-Hopf bifurcation. In Section 4, the generic bifurcations of codimension one and two are explored. A rich variety of solutions, including stationary, periodic and quasiperiodic (tori) are found. We present formulas for primary and secondary bifurcations of periodic solutions and tori, with the help of normal forms. In Section 5, the relevance of these results to the energy-transfer phenomenon of Nayfeh is considered, and directions of further research are indicated.

\section{Linear Analysis}

Definitions. Let $\mathbb{C}^{n^{2}}$ denote the set of all $n \times n$ complex matrices. A family A of matrices on the base $\mathcal{M}$ is a holomorphic mapping $A: \mathcal{M} \rightarrow \mathbb{C}^{n^{2}}$, where $\mathcal{M}$ is a neighbourhood of the origin in the parameter space $\mathbb{C}^{k}$. For brevity, we call this the family $A$. The germ of a family $A$ at the point $0 \in \mathcal{M}$ is called a deformation of the matrix $A(0)$. The elements $\mu \in \mathcal{M}$ are called parameters.

An example of a family of real matrices is given by $\mathbf{A}_{0}+\mathbf{C}(\hat{\mu})$ in equation (4) and a complex family is given by the linear part of (7). The algebra is simpler if we work with complex matrices. The real Jordan matrix $\mathbf{A}_{0}$ of (4) has the complex Jordan form

$$
\mathbf{A}_{c}=\left(\begin{array}{cccc}
0 & 1 & 0 & 0 \\
0 & 0 & 0 & 0 \\
0 & 0 & i & 0 \\
0 & 0 & 0 & -i
\end{array}\right)
$$

Two main questions are addressed in this section. The first question is how to find a canonical matrix, which is as simple as possible, and to which the linear part of all coupled-oscillator systems as described in Section 1 
can be reduced by a linear change of coordinates. An obvious candidate is the Jordan form; however, this is not a good choice because the reduction of a matrix to its Jordan form is not a stable operation when there are multiple eigenvalues, as is the case here. Although every member of a family containing $\mathbf{A}_{c}$ may be reduced to a Jordan form, the Jordan form does not in general depend continuously on the parameters, since there are matrices arbitrarily close to $\mathbf{A}_{c}$ for which the Jordan form is diagonal. The mapping which transforms matrices to Jordan form is discontinous. Therefore, we seek a different normal form, to which members of any family containing $\mathbf{A}_{c}$ may be transformed smoothly.

The second question relates to the choice of parameters $\mu \in \mathcal{M}$. What is the minimum set of parameters needed, and how can we know if any essential parameters are missing? In particular, are there systems with linear parts close to $\mathbf{A}_{0}$ which are not attainable by variations of the physical parameters in the given perturbation matrix $\mathbf{C}(\hat{\mu})$ in (4)? If we have in fact overlooked some essential parameters, then any study of the system in Section 1 is likely to miss important phenomena. We would like to have assurance that the parameter set in our family of matrices is sufficiently rich that all perturbations of $\mathbf{A}_{0}$ are included, and that there are no redundant parameters.

Both of these questions were answered by Arnold [3, 4, 5], who introduced a generalization of the Jordan form of a matrix $A$, with the minimum number of essential parameters, and to which all members of any family of $A$ can be transformed smoothly. This generalization of the Jordan normal form may be called the Arnold normal form of a matrix. The remainder of this section summarizes Arnold's construction of this normal form and shows how it answers the two questions above.

The reduction to Jordan form of any matrix $A$ is accomplished by a similarity transformation. Consider the Lie group $\mathrm{GL}(n, \mathbb{C})$ of all nonsingular matrices $S \in \mathbb{C}^{n^{2}}$. Then the group of similarity transformations on $\mathbb{C}^{n^{2}}$ is defined by the elements

$$
\operatorname{Ad}_{S}(A) \equiv S A S^{-1}, \quad A \in \mathbb{C}^{n^{2}}, S \in \operatorname{GL}(n, \mathbb{C}) .
$$

The group orbit of a given $A$ is

$$
\mathcal{O}(A)=\left\{\operatorname{Ad}_{S}(A)=S A S^{-1} \mid S \in \mathrm{GL}(n, \mathbb{C})\right\}
$$

and this is a smooth submanifold of $\mathbb{C}^{n^{2}}$. The orbit of $A$ thus consists of all matrices similar to $A$. It is well known that similar matrices share the 
same eigenvalues, with the same multiplicities and the same Jordan blocks (perhaps permuted). A convenient orbit representative for any given $A$ is its Jordan form, with Jordan blocks ordered in decreasing size, for example $\mathbf{A}_{c}$ in $(8)$.

Now, combining the concepts of deformations and similarity, we say that two deformations $B(\mu)$ and $A(\mu)$ of the same matrix $B(0)=A(0)=A_{0}$ are equivalent if there exists a deformation $C(\mu)$ of the identity matrix, for $\mu \in \mathcal{M}$, such that

$$
B(\mu)=C(\mu) A(\mu) C(\mu)^{-1} .
$$

Next, this equivalence is extended to allow two different parametrizations for $B$ and $A$. Let $\mathcal{M}$ and $\mathcal{N}$ be neighbourhoods of the origin in parameter spaces $\mathbb{C}^{k}$ and $\mathbb{C}^{l}$, respectively. Let $\varphi: \mathcal{M} \rightarrow \mathcal{N}$ be holomorphic at 0 , and $\varphi(0)=0$. Let $A(\alpha)$ be a family of matrices on $\mathcal{N}$. Define the family (deformation) induced from $A$ by $\varphi$ to be the family (deformation) $\varphi \circ A$

$$
(\varphi \circ A)(\mu)=A(\varphi(\mu))
$$

Definition. A deformation $A(\alpha)$ of $A_{0}$ is called versal if every deformation $B(\mu)$ of $A_{0}$ is equivalent to a deformation induced from $A(\alpha)$ :

$$
B(\mu)=C(\mu) A(\varphi(\mu)) C^{-1}(\mu), \quad C(0)=I, \varphi(0)=0 .
$$

A versal deformation is universal if mapping $\varphi$ is determined uniquely by deformation $B(\mu)$. It is miniversal if the dimension of the parameter space $\mathcal{N}$ is minimal among all versal deformations. This minimum dimension is called the codimension of the matrix $A_{0}$. The parameters in a miniversal deformation are also called unfolding parameters, and a (uni, mini-)versal deformation is sometimes called a (uni, mini-)versal unfolding.

It is clear that such a versal deformation exists and an upper bound on the codimension is $n^{2}$, since one could take as parameters all the elements of an $n \times n$ matrix. A lower bound is given by $n$, for the case of distinct eigenvalues and a diagonal Jordan form. In general, we need a manifold transversal to the group orbits. To this end, compute tangents to the group orbits in (10), for fixed $A=A_{0}$. Write $\psi(S) \equiv \operatorname{Ad}_{S}\left(A_{0}\right)=S A_{0} S^{-1}$, then the derivative with respect to $S$ at the identity $I$, for fixed $A_{0}$, is easily found to be the linear operator defined by commutation with $A_{0}$

$$
D \psi(I): \mathbb{C}^{n^{2}} \rightarrow \mathbb{C}^{n^{2}}, \quad D \psi(I) S=\left[S, A_{0}\right] \equiv S A_{0}-A_{0} S
$$


Definition. The centralizer $Z_{A}$ of matrix $A \in \mathbb{C}^{n^{2}}$ is the set of all matrices which commute with $A$

$$
\mathrm{Z}_{A}=\{B \mid[B, A] \equiv B A-A B=0\} .
$$

Thus, the nullspace of $D \psi(I)$ is the centralizer of $A_{0}$. The rank of $D \psi(I)$ is the dimension of the orbit of $A_{0}$. Since $D \psi(I)$ is square, the dimension of the centralizer is the codimension of the orbit, which is the minimum number of parameters in a versal deformation. This codimension was given by Arnold [3] in the following formula

$$
d=\sum_{i=1}^{s} \sum_{j=1}^{l_{i}}(2 j-1) n_{i j}\left(\lambda_{i}\right)
$$

where $\lambda_{i}, i=1, \ldots, s$ are distinct eigenvalues of $A_{0}, l_{i}$ is the number of Jordan blocks of $\lambda_{i}$ and $n_{i 1}\left(\lambda_{i}\right) \geq \cdots \geq n_{i l_{i}}\left(\alpha_{i}\right)$ are the corresponding orders of the Jordan blocks for each $\lambda_{i}$.

Furthermore, with respect to the Hermitian scalar product $\langle A, B\rangle=$ $\operatorname{tr}\left(A B^{*}\right)$, the orthogonal complement of the tangent to the orbit of $A_{0}$ in the tangent space $\mathbb{C}^{n^{2}}$ is given by $\left\{B^{*} \mid B \in \mathrm{Z}_{A_{0}}\right\}$. Here $B^{*}$ denotes the conjugate transpose of $B$. This is the form that the Fredholm Alternative takes in this setting. From this, Arnold obtains explicitly the versal deformations, see $[3,4,5,32]$.

Applying Arnold's method to the Jordan matrix $\mathbf{A}_{c}$ above gives the centralizer $\mathbf{Z}_{\mathbf{A}_{c}}=C(\alpha)$ and versal deformation $\mathbf{A}_{c}(\alpha)$ :

$$
\begin{gathered}
C(\alpha)=\left(\begin{array}{cccc}
\alpha_{2} & \alpha_{1} & 0 & 0 \\
0 & \alpha_{2} & 0 & 0 \\
0 & 0 & \alpha_{3} & 0 \\
0 & 0 & 0 & \alpha_{4}
\end{array}\right) \\
\mathbf{A}_{c}(\alpha)=\left(\begin{array}{cccc}
\alpha_{2} & 1 & 0 & 0 \\
\alpha_{1} & \alpha_{2} & 0 & 0 \\
0 & 0 & i+\alpha_{3} & 0 \\
0 & 0 & 0 & -i+\alpha_{4}
\end{array}\right) .
\end{gathered}
$$

Here $\alpha_{j}, j=1, \ldots, 4$ are arbitrary complex parameters. This versal deformation is not unique. Rather than the above orthogonal complement to the 
tangent space, we could choose any transversal subspace of minimum dimension, and obtain a miniversal deformation. One convenient choice replaces the $\alpha_{2}$ entry in the upper left corner of $\mathbf{A}_{c}(\alpha)$ by 0 , and thus reduces the number of nonzero entries. We adopt this choice.

Furthermore, since our original system is real, we decomplexify $\mathbf{A}_{c}(\alpha)$. The result of decomplexification (for this choice) is the real family of matrices

$$
\mathbf{A}_{0}(\alpha)=\left(\begin{array}{cccc}
0 & 1 & 0 & 0 \\
\alpha_{1} & \alpha_{2} & 0 & 0 \\
0 & 0 & \alpha_{3} & -1-\alpha_{4} \\
0 & 0 & 1+\alpha_{4} & \alpha_{3}
\end{array}\right)
$$

where $\alpha_{j}, j=1, \ldots, 4$ have been redefined as real parameters. We call the family in equation (17) the real Arnold normal form of the original matrix $\mathbf{A}_{0}$. It has the desired versality property, that any deformation $B(\mu)$ of $\mathbf{A}_{0}$ is equivalent to a deformation induced from the family $\mathbf{A}_{\alpha}$. In other words, any such $B(\mu)$ can be obtained by means of holomorphic mappings of parameters combined with similarity transformations, as given in (12). Among all such versal deformations, it has the minimum number of parameters.

Finally, we recall that our matrix is the linear part of a differential equation, in which a rescaling of time has the effect of multiplying the matrix by a positive real number. We choose to rescale time to keep the imaginary part of the complex eigenvalue equal to $\pm i$. This has the effect of making $\alpha_{4}=0$ in (17) and rescaling the other parameters. Therefore, in this context the codimension is 3 , and the versal deformation is

$$
\mathbf{A}_{0}(\alpha)=\left(\begin{array}{cccc}
0 & 1 & 0 & 0 \\
\alpha_{1} & \alpha_{2} & 0 & 0 \\
0 & 0 & \alpha_{3} & -1 \\
0 & 0 & 1 & \alpha_{3}
\end{array}\right)
$$

where now $\alpha_{j}, j=1,2,3$ represent redefined real unfolding parameters.

It remains to determine the mapping in (12) between the unfolding parameters $\alpha_{j}$ in Arnold's versal normal form (18) and the physical parameters in the original system (4). This may be done by comparing coefficients of the characteristic polynomials. The relationship is found to be

$$
\begin{aligned}
& \alpha_{1}=-\Omega+\cdots \\
& \alpha_{2}=-\delta_{1}+\cdots \\
& \alpha_{3}=-\frac{1}{2} \delta_{2}+\cdots
\end{aligned}
$$


where $\cdots$ represents higher order terms. Since this mapping is invertible at the origin, we have proven that the original family in (4), obtained via physical arguments, in fact yields a versal deformation.

The 3-parameter family of matrices (18) is the appropriate starting point for study of the phenomena associated with coupled oscillators near $0: 1$ resonance. As observed by Arnold in [5, page 221]:

... Then the natural object of study is not the individual object (say, a vector field with a complicated singular point), but a family so large that the singularity of the type under consideration does not disappear under a small deformation of the family. This simple argument of Poincaré shows the futility of such a large number of studies in the theory of differential equations and in other areas of analysis that it is always somewhat dangerous to mention it.

\section{Poincaré Normal Form}

The Poincaré normal form may be thought of as an extension of the ideas of the previous section from the linear case to the case of nonlinear vector fields. Arnold gives an excellent account of the theory and computation of the Poincaré normal form in [5]. Other useful references are [7, 9, 10, 14, 15, $17,18,23,32]$.

The basic idea of Poincaré is to to perform a sequence of near-identity transformations of the form

$$
\mathbf{x}=\mathbf{y}+\mathbf{h}_{k}(\mathbf{y}), \quad k=2,3, \ldots
$$

where $\mathbf{h}_{k}$ is a homogeneous polynomial of degree $k$. For each $k, \mathbf{h}_{k}$ is chosen to eliminate as many as possible of the terms of degree $k$ in the vector field. Terms which remain lie in a complement to the tangent space, at the identity, of the manifold of group orbits corresponding to (20); just as in the linear case of Section 2. The Poincaré normal form is not unique, since it depends on this choice of complement. Furthermore, the resulting power series does not converge in general; nor does the sequence of near-identity transformations (20) converge. This lack of convergence is often not a problem, since in many cases the phenomena of interest are determined by a finite truncation of the Poincaré normal form, of low degree. 
For the system of differential equations of interest in this paper as given by (7), the Poincaré normal form can be chosen to have a normal form symmetry [15], and can be written (formally) as

$$
\left(\begin{array}{c}
\dot{y}_{1} \\
\dot{y}_{2} \\
\dot{z}
\end{array}\right)=\left(\begin{array}{ccc}
0 & 1 & 0 \\
0 & 0 & 0 \\
0 & 0 & i
\end{array}\right)\left(\begin{array}{c}
y_{1} \\
y_{2} \\
z
\end{array}\right)+\left(\begin{array}{l}
\phi_{2} y_{1} \\
\phi_{1} y_{1}+\phi_{2} y_{2} \\
{\left[\phi_{3}+i \phi_{4}\right] z}
\end{array}\right)
$$

to which may be added the conjugate of the last equation, for $\dot{\bar{z}}$. Here $\phi_{i}, i=1, \ldots, 4$, are real valued formal power series (which may not converge) in the Hilbert basis of invariant polynomials $\left\{y_{1}^{2}, z \bar{z}=y_{3}^{2}+y_{4}^{2}\right\}$, i.e.

$$
\phi_{i}=\phi_{i}\left(y_{1}^{2}, z \bar{z}\right), \quad i=1, \ldots, 4 \text {. }
$$

Notice the formal resemblance between (21) and the complex Arnold normal form in the linear case, given by (16). In fact, if we were to allow the terms involving $\phi_{j}$ in the vector on the right-hand side of (21) to have linear parts, then the linear part of the Poincaré normal form would correspond to the Arnold normal form of its linearization.

The Poincaré normal form (21) can be further simplified by the nearidentity transformation:

$$
\left(y_{1}, y_{2}, z\right) \rightarrow\left(y_{1}, y_{2}+y_{1} \phi_{2}, z\right)
$$

which eliminates the nonlinear terms in the $\dot{y}_{1}$ equation of $(21)$. Now, the Poincaré normal form matches the Arnold normal form as given in (17), in the sense that we can identify

$$
\alpha_{j}=\phi_{j}(0,0), j=1, \ldots, 4
$$

Combining in one equation the versal deformation of the linearization, i.e. Arnold normal form (18), and this simplified Poincaré normal form, yields

$$
\left(\begin{array}{c}
\dot{y}_{1} \\
\dot{y}_{2} \\
\dot{z}
\end{array}\right)=\left(\begin{array}{ccc}
0 & 1 & 0 \\
\alpha_{1} & \alpha_{2} & 0 \\
0 & 0 & i+\alpha_{3}
\end{array}\right)\left(\begin{array}{c}
y_{1} \\
y_{2} \\
z
\end{array}\right)+\left(\begin{array}{c}
0 \\
\phi_{1} y_{1}+\phi_{2} y_{2} \\
{\left[\phi_{3}+i \phi_{4}\right] z}
\end{array}\right)
$$

where $y_{1}, y_{2} \in \mathbb{R}, z \in \mathbb{C}$, and $\phi_{j}=\phi_{j}\left(y_{1}^{2}, z \bar{z}\right), \phi(0,0)=0, j=1, \ldots, 4$, and we have rescaled time to make $i \alpha_{4}=0$. 
Finally, we decomplexify the $z$-equation to real form, but in polar rather than cartesian coordinates, to better exploit the normal form symmetry of the Hopf bifurcation. Let $z=r e^{i \theta}$. Then (22) can be written

$$
\begin{aligned}
\dot{y}_{1} & =y_{2} \\
\dot{y}_{2} & =\left[\alpha_{1}+\phi_{1}\right] y_{1}+\left[\alpha_{2}+\phi_{2}\right] y_{2} \\
\dot{r} & =\left[\alpha_{3}+\phi_{3}\right] r \\
\dot{\theta} & =1+\phi_{4} .
\end{aligned}
$$

Note that $\theta$ does not appear in the right hand sides of equations (23), so the $\dot{\theta}$-equation is decoupled from the other three. This is due to the $S^{1}$ symmetry of the normal form, induced by the Andronov-Hopf bifurcation $\mathrm{H}_{1}$, and it effectively reduces the problem from four dimensions to three. Given a solution $\left(y_{1}(t), y_{2}(t), r(t)\right)$ of the first three equations of (23), then the solution $\theta(t)$ is given by

$$
\theta(t)=\theta_{0}+t+\int_{0}^{t} \phi_{4}\left(y_{1}^{2}(s), r^{2}(s)\right) d s .
$$

All but exotic behaviour of this system is already determined by a cubic truncation of the Poincaré normal form. This can be written

$$
\begin{aligned}
\dot{y}_{1} & =y_{2} \\
\dot{y}_{2} & =\left[\alpha_{1}+\phi_{110} y_{1}^{2}+\phi_{101} r^{2}\right] y_{1}+\left[\alpha_{2}+\phi_{210} y_{1}^{2}+\phi_{201} r^{2}\right] y_{2} \\
\dot{r} & =\left[\alpha_{3}+\phi_{310} y_{1}^{2}+\phi_{301} r^{2}\right] r
\end{aligned}
$$

where $\phi_{j k l}$ is the coefficient, in the power series expansion of $\phi_{j}\left(y_{1}^{2}, r^{2}\right)$, of the term $y_{1}^{2 k} r^{2 l}$. The $\theta$ equation has been omitted in (24), but has a similar expansion. The system (24) is explored in the next section.

As has been shown in $[20,21]$, in special cases the higher order "tail" of the normal form can dramatically change the dynamics from the behaviour of a truncated normal form such as (24), since the tail breaks the normal form symmetry which led to the decoupling of the $\dot{\theta}$ equation. For example, when (24) has homoclinic or heteroclinic orbits, then the tail effects can produce homoclinic tangles, "horseshoes" and chaotic dynamics. These considerations will not be pursued in this paper.

\section{Bifurcations of Codimension One and Two}

The collection of all possible phase portraits of this system near the codimension three bifurcation point, or even of all the equivalence classes with respect 
to topological equivalence, is too large to list here. However, the stratified subvariety [5, page 227] of the bifurcation diagram in the parameter space $\mathbb{R}^{3}$ is relatively simple, and is presented in this section.

The stability of the trivial equilibrium solution is determined by the eigenvalues of the linear part $\mathbf{A}_{0}(\alpha)$, which are easily seen to be

$$
\lambda_{1,2}=\frac{\alpha_{2}}{2} \pm \sqrt{\alpha_{1}+\left(\frac{\alpha_{2}}{2}\right)^{2}}, \quad \lambda_{3,4}=\alpha_{3} \pm i
$$

All four eigenvalues have negative real part if and only if $\alpha_{j}, j=1,2,3$ are all negative. Therefore, the trivial equilibrium solution is asymptotically stable inside the negative octant of the parameter space $\mathcal{N} \in \mathbb{R}^{3}$, see Figure 1. As the point $\left(\alpha_{1}, \alpha_{2}, \alpha_{3}\right)$ leaves the negative octant, bifurcations occur. These bifurcations from the trivial equilibrium solution are called primary bifurcations. There are seven possibilities, see Figure 1: a point may leave by crossing through one of the three coordinate planes (codimension one), through one of the three coordinate axes (codimension two) or through the origin (codimension three). A generic exit is through a plane, codimension one. However, interesting and important mode-interactions occur around the intersections of the planes. These are best understood by studying the codimension two bifurcations on the coordinate axes. All six of these codimension one and two bifurcations have been studied in general, and are well understood. Their manifestations in the present system are described in the following subsections.

\subsection{Primary Bifurcations of Codimension One}

The three primary bifurcations of codimension one are: an Andronov-Hopf bifurcation with frequency of order $O(1)$ which we label $\mathrm{H}_{1}$, an AndronovHopf bifurcation with small frequency $O\left(\left|\alpha_{1}\right|\right)$, labeled $\mathrm{H}_{0}$, and a pitchfork bifurcation labeled P; see Figure 1.

\subsubsection{Primary Hopf Bifurcation $\mathrm{H}_{1}$}

For the system (22), the $z$-plane is a two-dimensional invariant subspace, on which the normal form equations reduce to

$$
\begin{aligned}
y_{1}=y_{2} & =0 \\
\dot{r} & =\left[\alpha_{3}+\phi_{3}\left(0, r^{2}\right)\right] r \\
\dot{\theta} & =1+\phi_{4}\left(0, r^{2}\right) .
\end{aligned}
$$


This has the family of periodic solutions

$$
\begin{aligned}
y_{1}=y_{2} & =0 \\
r & =\sqrt{\frac{-\alpha_{3}}{\phi_{301}}}+\cdots \\
\theta(t) & =\theta_{0}+\left[1+\phi_{4}\left(0, r^{2}\right)\right] t(\bmod 2 \pi) .
\end{aligned}
$$

This is a classical Andronov-Hopf bifurcation, giving birth to a limit cycle in the $\left(y_{3}, y_{4}\right)$-plane with period near $2 \pi$ (angular frequency 1 ) as the parameter $\alpha_{3}$ crosses through 0 , provided $\alpha_{1,2} \neq 0$. Generically $\phi_{301} \neq 0$ and the bifurcation is nondegenerate, which is assumed to be the case in this paper; then the limit cycle is locally unique. Degenerate cases of Hopf bifurcation are explored in detail in [13]. If $\phi_{301}<0$, then the bifurcation is supercritical (the limit cycle exits for $\alpha>0$ ) and if $\phi_{301}>0$ the bifurcation is subcritical (exists for $\alpha<0$ ). A supercritical limit cycle is stable if $\alpha_{1,2}<0$, otherwise the cycle is unstable.

\subsubsection{Pitchfork Bifurcation $\mathrm{P}$}

Any equilibrium solution of (22) lies on the $y_{1}$ axis, and satisfies

$$
\left[\alpha_{1}+\phi_{1}\left(y_{1}^{2}, 0\right)\right] y_{1}=0
$$

This is a classic pitchfork bifurcation, yielding in addition to the trivial solution $\mathbf{y}=0$, a symmetric pair of nontrivial solutions

$$
y_{1}= \pm \sqrt{-\frac{\alpha_{1}}{\phi_{110}}}+\cdots
$$

branching from 0 as $\alpha_{1}$ crosses through zero, provided $\phi_{110} \neq 0$ and $\alpha_{2,3} \neq 0$, which we assume. The pitchfork is supercritical if $\phi_{110}<0$ and subcritical if $\phi_{110}>0$. Supercritical solutions are stable if $\alpha_{2,3}<0$, otherwise both are unstable.

\subsubsection{Primary Hopf Bifurcation $\mathrm{H}_{0}$}

The Poincaré normal form $(22)$ leaves the $\left(y_{1}, y_{2}\right)$-plane invariant. On this plane, the normal form reduces to

$$
\begin{aligned}
\dot{y}_{1} & =y_{2} \\
\dot{y}_{2} & =\left[\alpha_{1}+\phi_{1}\left(y_{1}^{2}, 0\right)\right] y_{1}+\left[\alpha_{2}+\phi_{2}\left(y_{1}^{2}, 0\right)\right] y_{2} \\
z & =0
\end{aligned}
$$


For fixed $\alpha_{1}<0$, this system has an Andronov-Hopf bifurcation as $\alpha_{2}$ crosses through zero, for any $\alpha_{3} \neq 0$. The new limit cycle has a small frequency, asymptotic to $\sqrt{-\alpha_{1}}$. The change of variables $x=y_{1}, y=-y_{2} / \omega$ where $\omega^{2}=-\alpha_{1}$ transforms (29) to the planar system

$$
\left(\begin{array}{l}
\dot{x} \\
\dot{y}
\end{array}\right)=\left(\begin{array}{cc}
0 & -\omega \\
\omega & 0
\end{array}\right)\left(\begin{array}{l}
x \\
y
\end{array}\right)+\left(\begin{array}{c}
0 \\
-\frac{1}{\omega} \phi_{1}\left(x^{2}, 0\right) x+\phi_{2}\left(x^{2}, 0\right) y
\end{array}\right) .
$$

This planar system has the Andronov-Hopf normal form, with $x+i y=\rho e^{i \varphi}$

$$
\begin{aligned}
& \dot{\rho}=\left[\frac{\alpha_{2}}{2}+\frac{1}{8} \phi_{210} \rho^{2}+\cdots\right] \rho \\
& \dot{\varphi}=\sqrt{-\alpha_{1}-\left(\frac{\alpha_{2}}{2}\right)^{2}}+b \rho^{2}+\cdots .
\end{aligned}
$$

From this it follows that the limit cycle has amplitude given by

$$
\rho=\sqrt{-\frac{4 \alpha_{2}}{\phi_{210}}}+\cdots
$$

and is super-(sub-)critical if $\phi_{210}<0(>0)$.

\subsection{Primary Bifurcations of Codimension Two}

The Poincaré normal form (22) has three bifurcations of codimension two from the trivial solution in state space. In the parameter space they occur along the three coordinate axes, see Figure 1.

\subsubsection{Bogdanov-Takens Bifurcation BT}

As observed for equation (29), the $\left(y_{1}, y_{2}\right)$-plane is invariant for the flow of the normal form. On this plane, for $\alpha_{3} \neq 0$ and $\left(\alpha_{1}, \alpha_{2}\right)$ in a neighbourhood of $(0,0)$, the system has a $\mathbb{Z}_{2}$-symmetric Bogdanov-Takens bifurcation $[8,31]$, see line BT in Figure 1. It yields stable solutions only if $\alpha_{3}<0$. In addition to the codimension one primary bifurcations $\mathrm{P}$ and $\mathrm{H}_{0}$ described above, there are rich possibilities including simultaneous secondary Andronov-Hopf bifurcations from the pitchfork pair of equilibria, coalescence of two limit cycles, orbits homoclinic to the origin and heteroclinic orbits joining the two pitchfork equilibria. A detailed description of the versal deformations, bifurcation diagrams and phase portraits is given by Arnold [5, page 300], and need not be repeated here. 
New possibilities arise in this system via the interaction of the BogdanovTakens bifurcation with the primary Andronov-Hopf bifurcation $\mathrm{H}_{1}$, leading to a flow in a phase space which is essentially $\mathbb{R}^{2} \times \mathrm{S}^{1}$. The simple homoclinic orbits of the planar Bogdanov-Takens bifurcation then lead to transversally intersecting two-dimensional stable and unstable manifolds of periodic orbits and thus to chaotic dynamics, as for example in [14, 23, 32]. These phenomena merit further investigation.

\subsubsection{Pitchfork-Hopf Mode Interaction PH}

In a neighbourhood of the $\alpha_{2}$-axis for $\alpha_{2} \neq 0$, the normal form (22) has a pitchfork-Hopf mode interaction, see PH in Figure 1. On the $\alpha_{2}$-axis, the system has a simple zero eigenvalue, with a $\mathbb{Z}_{2}$-symmetry on the corresponding eigenspace $y_{1}$, and a pair of purely imaginary eigenvalues $\pm i$.

Near $\left(\alpha_{1}, \alpha_{3}\right)=(0,0)$ with $\alpha_{2}<0$, one finds the expected pitchfork and Hopf primary bifurcations $\mathrm{P}$ and $\mathrm{H}_{1}$, and additional mixed-mode solutions due to the nonlinear coupling of these primary bifurcations. A complete classification of nondegenerate pitchfork-Hopf mode interactions is given in [22]. To investigate these here, in (23) we set $\dot{y}_{1}=y_{2}=0, \dot{r}=0$, suppress the $\dot{\theta}$ equation and retain only leading order terms in $\phi_{1}$ and $\phi_{3}$. Then equation (24) reduces to

$$
\begin{aligned}
& 0=\left[\alpha_{1}+\phi_{110} y_{1}^{2}+\phi_{101} r^{2}\right] y_{1} \\
& 0=\left[\alpha_{3}+\phi_{310} y_{1}^{2}+\phi_{301} r^{2}\right] r
\end{aligned}
$$

For nondegeneracy, we assume

$$
\Delta_{1} \equiv \phi_{110} \phi_{301}-\phi_{310} \phi_{101} \neq 0, \quad \phi_{110} \phi_{301} \neq 0 \text {. }
$$

Then (32) has four branches of solutions

$$
\begin{aligned}
\left(y_{1}^{2}, r^{2}\right) & =(0,0) \\
\left(y_{1}^{2}, r^{2}\right) & =\left(-\frac{\alpha_{1}}{\phi_{110}}, 0\right) \\
\left(y_{1}^{2}, r^{2}\right) & =\left(0,-\frac{\alpha_{3}}{\phi_{301}}\right) \\
\left(y_{1}^{2}, r^{2}\right) & =\left(\frac{\alpha_{3} \phi_{101}-\alpha_{1} \phi_{301}}{\Delta_{1}}, \frac{\alpha_{1} \phi_{310}-\alpha_{3} \phi_{110}}{\Delta_{1}}\right) .
\end{aligned}
$$


Note that the nonzero quantities on the right must be positive for the solutions to be real. The first three of these solutions are the previously identified trivial, primary $\mathrm{P}$ and primary $\mathrm{H}_{1}$ solutions. The fourth is a mixed-mode solution, which links the $\mathrm{P}$ and $\mathrm{H}_{1}$ solutions, at the two ends of its interval of (real) existence, in secondary bifurcations. Due to symmetry, the primary bifurcations have two symmetric branches $\pm y_{1}, \pm r$, and the secondary bifurcations would seem to have four branches; however, in the full dynamics the solutions with $\pm r$ give the same periodic orbit with a phase shift. The secondary bifurcation from $\mathrm{P}$ is an Andronov-Hopf bifurcation, while the secondary bifurcation from $\mathrm{H}_{1}$ is a pitchfork bifurcation of two new limit cycles.

While all of the above results were deduced using only the cubic truncation (32), they extend by standard arguments to the full equations, using assumptions (33), the Implicit Function Theorem and transversality. When $\phi_{110} \phi_{301}<0$ and higher order terms are included, it is possible to show that the phase portraits may include also a flow on a two-dimensional invariant torus with a small second frequency. The effects of the symmetry-breaking normal form tail may then lead to chaos, see [14, 16, 19, 20, 21].

\subsubsection{Hopf-Hopf Mode Interaction $\mathrm{HH}$}

In a neighbourhood of the negative $\alpha_{1}$ semi-axis (see HH in Figure 1), nonresonant Hopf-Hopf mode interactions occur, involving the $\mathrm{H}_{1}$ and $\mathrm{H}_{0}$ bifurcations. For $\left(\alpha_{2}, \alpha_{3}\right)=(0,0)$, the full system has two pairs of purely imaginary eigenvalues, which are nonresonant because the frequency ratio is very small. The four-dimensional system can be written as two pairs of amplitude-phase equations, in $(r, \theta)$ and $(\rho, \varphi)$, corresponding to the two primary AndronovHopf bifurcations $\mathrm{H}_{1}$ and $\mathrm{H}_{0}$ respectively. The two phase equations decouple due to nonresonance, leaving two amplitude equations which have the $\mathbb{Z}_{2} \oplus \mathbb{Z}_{2}$ symmetric normal form

$$
\begin{aligned}
& \dot{r}=\left[\alpha_{3}+c \rho^{2}+\phi_{301} r^{2}+\cdots\right] r \\
& \dot{\rho}=\left[\frac{1}{2} \alpha_{2}+\frac{1}{8} \phi_{210} \rho^{2}+d r^{2}+\cdots\right] \rho .
\end{aligned}
$$

This has four families of solutions satisfying $(\dot{r}, \dot{\rho})=(0,0)$, given to leading order by

$$
(r, \rho)=(0,0)
$$




$$
\begin{aligned}
& (r, \rho)=\left(\sqrt{-\frac{\alpha_{3}}{\phi_{301}}}, 0\right) \\
& (r, \rho)=\left(0, \sqrt{-\frac{4 \alpha_{2}}{\phi_{210}}}\right) \\
& (r, \rho)=\left(\sqrt{\frac{\frac{1}{2} \alpha_{2} c-\frac{1}{8} \alpha_{3} \phi_{210}}{\Delta_{2}}}, \sqrt{\frac{\alpha_{3} d-\frac{1}{2} \alpha_{2} \phi_{301}}{\Delta_{2}}}\right)
\end{aligned}
$$

where $\Delta_{2}=\frac{1}{8} \phi_{210} \phi_{301}-c d \neq 0$. The first three of these are the previously obtained trivial, $\mathrm{H}_{1}$ and $\mathrm{H}_{0}$ solutions, respectively. The fourth gives a new family of two-frequency solutions, on an invariant 2-torus. The local persistence of this invariant torus is guaranteed by standard arguments involving normal hyperbolicity and nonresonance. This torus appears through a secondary bifurcation from either of the primary Andronov-Hopf families. To leading order, the 2-torus bifurcates from the $\mathrm{H}_{1}$ periodic orbit when

$$
\alpha_{3} d=\frac{1}{2} \alpha_{2} \phi_{301}, \rho=0, r=\sqrt{-\frac{\alpha_{3}}{\phi_{301}}},
$$

and bifurcates from the $\mathrm{H}_{0}$ periodic orbit when

$$
\alpha_{2} c=\frac{1}{4} \alpha_{3} \phi_{210}, \quad \rho=\sqrt{-\frac{4 \alpha_{2}}{\phi_{210}}}, r=0 .
$$

Near $\mathrm{H}_{1}$, the solutions on the 2-torus are close to the $\mathrm{H}_{1}$ periodic orbit, with primary frequency $\mathcal{O}(1)$, but have an amplitude modulation with frequency $\mathcal{O}\left(\sqrt{-\alpha_{1}}\right)$. A description of possible dynamics in nonresonant Hopf-Hopf mode interactions is given in [14]. In certain cases, there is a further possibility of a stable 3-torus bifurcation from the 2-torus, and even

of chaotic dynamics [16]. This analysis requires consideration of higher order terms.

\section{Conclusions}

We may draw several conclusions from this study.

The first important conclusion is that the simultaneous occurrence of Andronov-Hopf and Bogdanov-Takens bifurcations is a codimension-three phenomenon, requiring three independent parameters for its exploration and 
understanding. The three parameters in Arnold's versal deformation may be chosen so that they directly determine the three primary bifurcations of codimension one: $\alpha_{1}$ determines the primary pitchfork bifurcation, $\alpha_{2}$ determines the primary Hopf bifurcation with low frequency (of order $\sqrt{-\alpha_{1}}$ ) and $\alpha_{3}$ determines the primary Hopf bifurcation with high frequency (of order 1). These three versal unfolding parameters are related to the physical parameters of the coupled oscillators by formulae (19).

In the versal deformation of the original codimension three bifurcation, there are primary bifurcations of six different types; three of codimension one and three of codimension two, all of which are understood. The codimension two bifurcations lead to secondary bifurcations, and produce new families of solutions which are linkages between primary bifurcation families.

The double zero eigenvalue may lead to a pitchfork bifurcation or a Hopf bifurcation of low frequency, and may lead to simultaneous existence of both nontrivial equilibria and low-frequency limit cycles, as in the standard Bogdanov-Takens analysis. When coupled with the primary Andronov-Hopf bifurcation $\mathrm{H}_{1}$, it may yield homoclinic chaos [32].

The nonlinear coupling between the primary high frequency and low frequency Andronov-Hopf bifurcations can lead to a quasiperiodic flow on a 2-torus, with its two frequencies close to those of the two primary AndronovHopf bifurcations. This gives a plausible explanation for the observations of Nayfeh et al. [28, 29, 30], of low frequency modulation of a high frequency mode, and of energy transfer from high to low frequency modes, near $0: 1$ resonance. The case studied by Nayfeh corresponds to a choice of unfolding parameters $\alpha_{1}<0$ (fixed) and $\alpha_{2}, \alpha_{3}$ both small and negative; i.e., near the negative $\alpha_{1}$ axis marked HH in Figure 1. This is the location where birth of a 2 -torus is expected, from the normal form analysis. A more detailed investigation of this particular application, using both analytical and numerical techniques, is in progress.

The present study provides indications of other directions for future research. Systems of coupled oscillators with less symmetry than required by (2) also are important in applications, and may be investigated by these same normal form methods. The effect of higher order terms, particularly in the onset of more complex dynamics, is worthy of investigation. As well, the application of these results to concrete systems in mechanical or electrical engineering, and other fields, may lead to new insights. 


\section{References}

[1] A.A. Andronov, A.A Vitt and S.E. Khaikin. Theory of Oscillators. Dover, New York, 1987.

[2] V.I. Arnold. Small denominators I. Mappings of the circumference onto itself. AMS Translations (2) 46 (1965) 213-284.

[3] V.I. Arnold. On matrices depending on parameters. Russian Mathematical Surveys 26 (1971) 29-43.

[4] V.I. Arnold. Lectures on bifurcations in versal families. Russian Mathematical Surveys 27 (1972) 54-123.

[5] V. I. Arnold. Geometrical Methods in the Theory of Ordinary Differential Equations. Springer-Verlag, New York, 1983.

[6] V. I. Arnold. Mathematical Methods of Classical Mechanics. Second edition, Springer-Verlag, New York, 1989.

[7] Yuri N. Bibikov. Local Theory of Nonlinear Analytic Ordinary Differential Equations. LNM 702, Springer-Verlag, New York, 1979.

[8] R. I. Bogdanov. Versal deformations of a singular point on the plane in the case of zero eigenvalues. Functional Analysis and Its Applications 9 (1975) 144-145.

[9] Alexander D. Bruno. Local Methods in Nonlinear Differential Equations. Springer-Verlag, Berlin 1989.

[10] S-N. Chow, C. Li and D. Wang. Normal forms and bifurcations of planar vector fields. Cambridge University Press, 1994.

[11] I. T. Georgiou, A. K. Bajaj and M. Corless. Slow and fast invariant manifolds, and normal modes in a two-degree-of-freedom structural dynamical system with multiple equilibrium states. Int. J. Non-Linear Mechanics 33 (1998) 275-300.

[12] S. A. van Gils, M.P. Krupa and W. F. Langford. Hopf bifurcation with nonsemisimple 1:1 resonance. Nonlinearity 3 (1990) 1-26. 
[13] M. Golubitsky and W. F. Langford. Classification and unfoldings of degenerate Hopf bifurcations. J. Diff. Eq. 41 (1981) 375-415.

[14] J. Guckenheimer and P. Holmes. Nonlinear Oscillations, Dynamical Systems and Bifurcations of Vector Fields. Springer-Verlag, New York, 1983.

[15] G. Iooss and M. Adelmeyer. Topics in Bifurcation Theory and Applications. World Sci., Singapore, 1992.

[16] G. Iooss and W. F. Langford. Conjectures on the routes to turbulence via bifurcations. In Nonlinear Dynamics, Annals N.Y. Acad. Sci. 357 (1980) 489-505.

[17] Peter B. Kahn and Yair Zarmi. Nonlinear Dynamics: Exploration through Normal Forms. Wiley-Interscience, New York, 1998.

[18] L. G. Khazin and E. E. Shnol. Stability of Critical Equilibrium States. Manchester Univ. Press, Manchester, 1991.

[19] W. F. Langford. Periodic and steady mode interactions lead to tori. SIAM J. Appl. Math. 37 (1979) 22-48.

[20] W. F. Langford. A review of interactions of Hopf and steady-state bifurcations. In Dynamical Systems and Turbulence. G.I. Barenblatt, G. Iooss and D.D. Joseph editors, Pitman, London, 1983, 215-237.

[21] W. F. Langford. Numerical studies of torus bifurcations. In Numerical Methods for Bifurcation Problems. T. Küpper, H.D. Mittelmann and H. Weber editors, ISNM 70 Birkhäuser Verlag, Boston, 1984, 285-294.

[22] W. F. Langford and G. Iooss. Interactions of Hopf and pitchfork bifurcations. In Bifurcation Problems and their Numerical Solution. H.D. Mittelmann and H. Weber editors, ISNM 54, Birkhäuser Verlag, Basel, 1980, 103-134.

[23] W. F. Langford and W. Nagata. Normal Forms and Homoclinic Chaos, Fields Institute Communications 4. American Mathematical Society, Providence, 1995. 
[24] Luo Dingjun, Wang Xian, Zhu Deming and Han Maoan. Bifurcation Theory and Methods of Dynamical Systems. World Scientific, Singapore, 1997.

[25] P. Moson. Quasi-periodic solutions of a special system. In Differential Equations: Qualitative Theory. North-Holland, 1984.

[26] P. Moson. Local bifurcations in the case of eigenvalues $0^{2}, \pm i$. Z. Angew. Math. Mech. 71 (1991) T69-T70.

[27] W. Nagata and N. Sri Namachchivaya. Bifurcations in gyroscopic systems with an application to rotating shafts. Proc. $R$. Soc. Lond. A 454 (1998) 543-585.

[28] S. A. Nayfeh and A. H. Nayfeh. Nonlinear interactions between two widely spaced modes - external excitation. International Journal of Bifurcation and Chaos 3 (1993) 417-427.

[29] S. A. Nayfeh and A. H. Nayfeh. Energy Transfer from high- to lowfrequency modes in a flexible structure via modulation. Journal of $\mathrm{Vi}$ bration and Accoustics 116 (1994) 203-207.

[30] A. H. Nayfeh, S. A. Nayfeh, T. A. Anderson and B. Balachandran. Transfer of energy from high-frequency to low-frequency modes. In Nonlinearity and Chaos in Engineering Dynamics. John-Wiley \& Sons, Chichester, 1994.

[31] F. Takens. Singularities of vector fields. Publ. Math. IHES. 43 (1974) 47-100.

[32] S. Wiggins. Introduction to Applied Nonlinear Dynamical Systems and Chaos. Springer-Verlag, New York, 1990.

[33] P. Yu and K. Huseyin. Bifurcations associated with a double zero and a pair of pure imaginary eigenvalues. SIAM J. Appl. Math. 48 (1988) 229-261. 


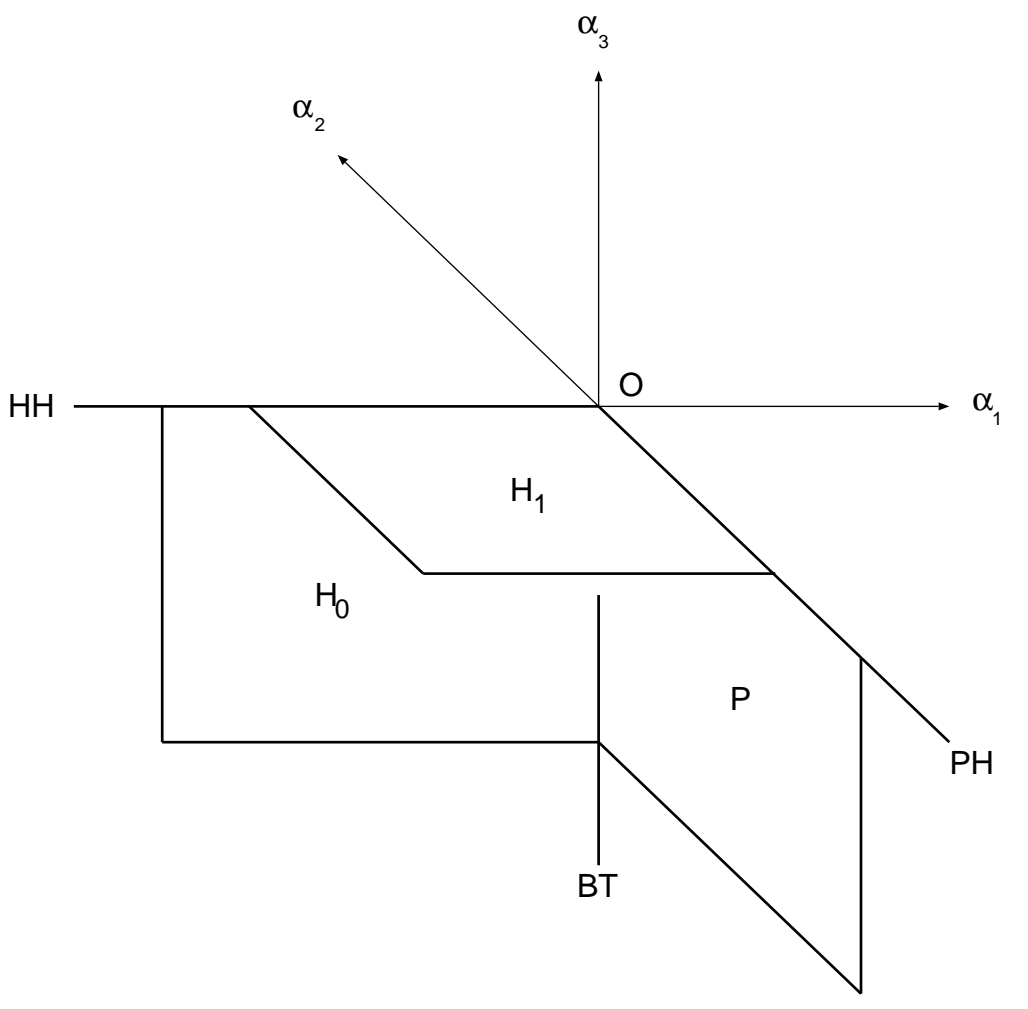

Figure 1: 